\title{
Kommentar
}

Schweiz. Zschr. GanzheitsMedizin 17, 332-334 (2005). @ Verlag für GanzheitsMedizin, Basel. www.ganzheitsmedizin.ch

\section{Andy Suter}

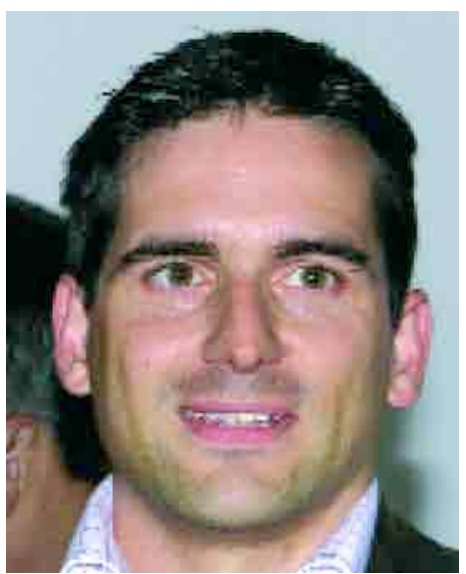

* Referat an der Ärzteforbildung «Ausgewählte phytotherapeutische Behandlungsansätze in Urologie und Rheumatologie",

19. / 20. August 2005, Roggwil.

\section{Sägepalmenextrakt kann mehr als nur Prostatabeschwerden lindern}

\author{
Resultate aus neuen in vitro Untersuchungen mit dem \\ Sägepalmenextrakt A. Vogel ProstaMed ${ }^{\circledR}$ *
}

$D^{\prime}$ e Sägepalme (Sabal serrulata, Serenoa repens) gehört zur Familie der Betelpalmgewächse und ist in den südlichen Küstenstaaten Nordamerikas (North Carolina, Florida) sowie in Mittel- und im tropischen Südamerika heimisch. Die niedrigstämmige Zwergpalme wächst aus einem kriechenden Wurzelstock und bildet bis zu 1.5 m lange Blattstiele. Die charakteristischen, fächerfö rmigen Blätter sind in bis zu 24 Segmente aufgeteilt. Träger der pharmakologisch wirksamen Inhaltsstoffe sind die olivengrossen, im getrockneten Zustand dunkelrot-bläulich bis schwarz gefärbten Beerenfrüchte [1].

Sie wurden von der indigenen Bevölkerung traditionell als Nahrungsund Heilmittel verwendet. Gemäss HALE [2] waren sie Grundnahrungsmittel der in Florida beheimateten Seminole-Indianer: Sie benutzten grösstenteils die Früchtetinktur und den zerdrückten Samen als Heilmittel zur Behandlung von Zystitis, Gonorrhö, Schleimhautreizungen und bei vergrösserter Prostata. Die Eingeborenen der mittel- und südamerikanischen Staaten verwendeten die Sägepalme als Arzneimittel mit diuretischen, tonisierenden und relaxierenden Eigenschaften. Beruhigend wirkt vor allem das gezuckerte Samenöl, das hauptsächlich bei Schlaflosigkeit, starkem Husten und Bronchitis eingesetzt wurde [3]. Während der heutige Anwendungsbereich auf die Behandlung der benignen Prostatahyperplasie (BPH) eingeschränkt ist, wurden Sägepalmenpräparationen ursprünglich vielfach auch bei Frauen mit urogenitalen Beschwerden eingesetzt, in der Literatur wird sogar der Einsatz als Aphrodisiakium bei beiden Geschlechtern beschrieben.

Neuere und viel versprechende Anwendungsgebiete für die Sägepalme, die durch erste, interessante Pilotstudien untermauert werden, sind die androgene Allopezie [4,5] und die Prostatitis [6], zudem weisen Einzelfallberichte auch auf eine gute Wirksamkeit bei dysurischen Beschwerden der Frau, wie der Reizblase, hin.

Diese Aufzählung der verschiedenen Indikationsgebiete der Sägepalme zeigt, dass ihr Einsatz nur bei der benignen Prostatahyperplasie, wie man ihn heute im westlichen Kulturkreis kennt, den Möglichkeiten dieser Heilpflanze nicht gerecht wird. Wir haben daher in drei in vitro-Untersuchungen den Sägepalmen-Extrakt A. Vogel ProstaMed ${ }^{\circledR}$ untersucht, um eine erste wissenschaftliche Grundlage für den erweiterten Einsatz in anderen Indikationsgebieten zu erhalten. Im Folgenden sind die bisher unveröffentlichten Daten zusammenfassend beschrieben.

\section{Antibakterielle Wirksamkeit}

In einer umfassenden Untersuchung wurden unter der Leitung von Prof. Jürgen Reichling, Ruprecht-KarlsUniversität in Heidelberg, Keime, die im Urogenitaltrakt des Menschen Infektionen herv o rrufen können, getestet. Gerade bei Prostataerkrankungen mit Miktionsbeschwerden besteht durch das andauernde Verbleiben von 
Tab. 1. Minimale Hemmkonzentration und minimale bakterizide Konzentration von Sägepalmenextrakt bei verschiedenen Bakterienstämmen

\begin{tabular}{|lcc|}
\hline & $\begin{array}{l}\text { Minimale bakterizide } \\
\text { Konzentration [\% V/V] }\end{array}$ & $\begin{array}{c}\text { Minimale Hemm- } \\
\text { konzentration [\% V/V] }\end{array}$ \\
grampositiv & 0.125 & 0.25 \\
Staphylococcus aureus & 0.25 & 0.5 \\
Staphylococcus aureus & 2 & $>2$ \\
methicillinresistent & $0.25-0.125$ & 1 \\
Staphylococcus epidermis & $1-0.5$ & 2 \\
Staphylococcus saprophyticus & $0.25-0.125$ & $0.5-0.25$ \\
Enterococcus faecalis & & $>2$ \\
Lysteria monocytogenes & $>2$ & $>2$ \\
gramnegativ & $>2$ & $>2$ \\
Escherichia coli & $>2$ & \\
Klebsiella pneumoniae & & \\
Proteus mirabilis & & \\
\hline
\end{tabular}

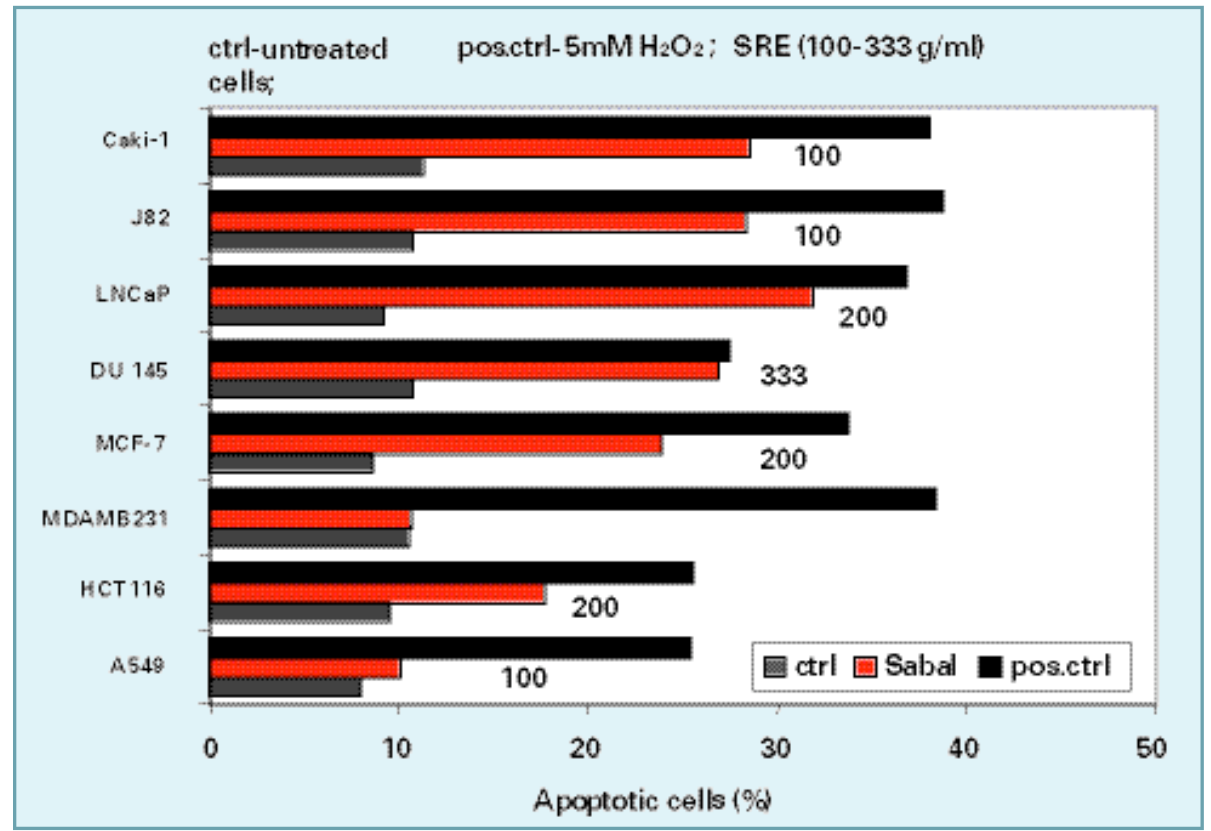

Abb. 1. Apoptose-Induktion nach 24h Inkubation durch den Sägepalmeextrakt A. Vogel ProstaMed® in verschiedenen karzinogenen Zelllinien (Caki-1: Nieren-Ca; J82: Blasen-Ca; LNCaP: Prostata Ca; DU 145: Prostata Ca; MCF-7: Mamma Ca (Estr+); MDAMB231: Mamma Ca (Estr-); HCT116:Colon $\mathrm{Ca}$; A549: Lungen Ca).

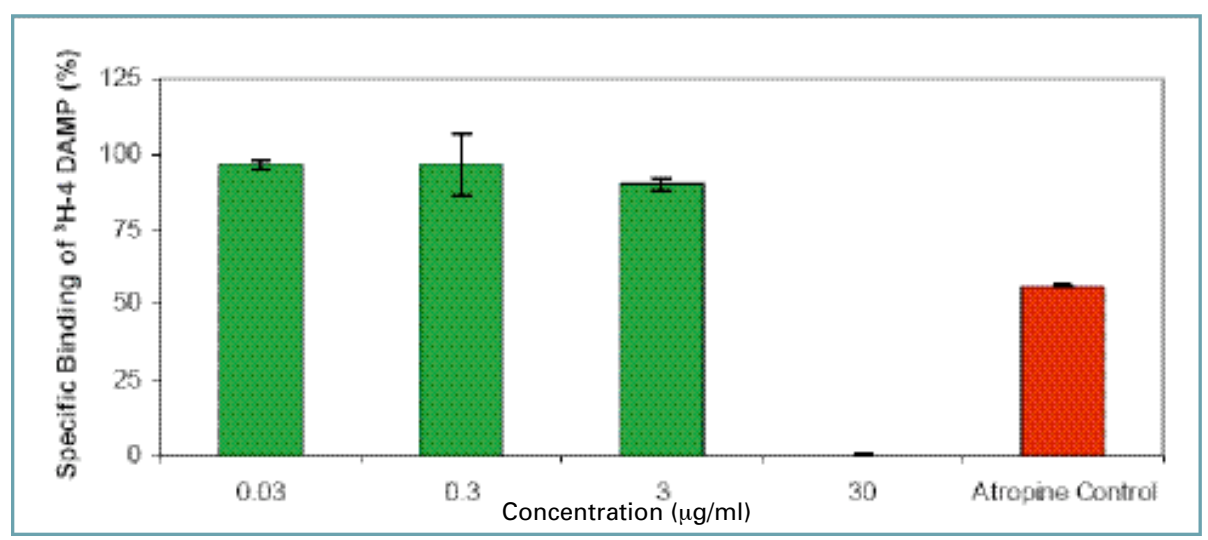

Abb. 2. Verdrängung der für den muskarinischen Rezeptor M3 spezifischen Substanz DAMP durch Sägepalmenextrakt mit einer IC50 zwischen 3-30 $\mu \mathrm{g} / \mathrm{ml}$.
Restharn in der Blase eine erhöhte Infektionsgefahr. Harnwegsinfektionen hingegen werden meist durch Bakterien hervorgerufen, die normalerweise die Haut im Anal- und Genitalbereich sowie die Darm- und Vaginalschleimhaut besiedeln, aber bei Schwächung des Immunsystems und/ oder falscher Körperhygiene in den ableitenden Harnwegen aufsteigen können. Der häufigste Verursacher unkomplizierter Harnwegsinfektionen ist E.coli neben weiteren gramnegativen Enterobakterien und grampositiven Enterokokken und Staphylokokken. Tabelle 1 zeigt die minimale Hemmkonzentration (MHK) und die bakterizide Hemmkonzentration von A. Vogel ProstaMed ${ }^{\circledR}$ bei den getesteten Bakterienstämmen. Der Extrakt ProstaMed ${ }^{\circledR}$ zeigte gegenüber grampositiven Keimen in deutlich geringerer Konzentration eine antimikrobielle Wirksamkeit als gegenüber gramnegativen Keimen. Interessant sind die MHK gegenüber $S$. saprophyticus, $S$. aureus und vor allem gegenüber methilinresistentem $S$. aureus, der heute gerade in Spitälern ein Problem darstellt. Die MHK von A. Vogel ProstaMed ${ }^{\circledR}$ liegen hier im Bereich ätherischer Öle, wie z.B. Teebaumöl.

\section{Apoptotische Wirksamkeit}

Da Sägepalmenextrakt bei der Behandlung der BPH chronisch eingenommen wird, stellt sich die Frage, welchen zusätzlichen positiven Effekt diese Langzeitbehandlung auch noch mit sich ziehen kann. Bei synthetischen Präparaten zur Behandlung der BPH wird eine chemopräventive Wirkung diskutiert, daher wurde unter der Leitung von Prof. Dr. Reinhard Saller und Dr. Katarina Hostanska, Institut für Naturheilkunde der Universität Zürich, die direkte apoptotische Wirkung von A. Vogel ProstaMed ${ }^{\circledR}$ auf verschiedene Krebszelllinien untersucht.

Neben zwei Prostatakarzinomzelllinien wurden zusätzlich sechs weitere Karzinomzelllinien getestet, da mit der systemischen Verfügbarkeit des Extraktes auch eine Wirkung auf andere Organe als die des Urogenitaltraktes denkbar wäre. In Abbildung 1 ist ersichtlich, dass der Extrakt durch- 
schnittlich etwa $30 \%$ der Zellen nach einer Behandlungsdauer von 48 Stunden durch einen programmierten Zelltod absterben liess (unveröffentlichte Daten). Auch wenn die Extrapolation auf die In-vivo-Situation sicher schwierig ist, zeigen diese Resultate doch, dass Patienten, die Sägepalmenextrakt während längerer Zeit zu sich nehmen, zusätzlich von einem chemopräventiven Effekt profitieren können.

\section{Einfluss auf Rezeptoren des Urogenitaltraktes und auf den Testosteronhaushalt}

Zur Verbesserung der dynamischen Komponente einer BPH werden gerade in der Anfangsphase der Behandlung gerne Alpha-Blocker eingesetzt. Diese Medikamente blockieren die $\alpha 1$ Rezeptoren im Bereich des Harnblasenhalses und der Urethra und können somit die intravesikale Obstruktion verringern und $\mathrm{zu}$ einer Verbesserung der Symptomatik führen.

Bei Inkontinenzbeschwerden der Frau werden heute zudem auch Anticholinergika, die gezielt die muskarinischen Rezeptoren blockieren, und Serotonin-Wiederaufnahmehemmer eingesetzt. Zudem wird postuliert, dass eine erhöhter Testosteronspiegel bei weiblichen Patienten eine positive Wirkung auf die Stärkung der Blasenmuskulatur haben könnte.

Daher wurde in verschiedenen Invitro-Modellen untersucht, wie stark A. Vogel ProstaMed ${ }^{\circledR}$ den $\alpha 1$-Adrenorezeptor und verschiedene muskarinische Rezeptoren zu hemmen vermag und ob ein Einfluss auf den Serotonin-
Transporter und den Testosteronhaushalt nachweisbar sind.

Während kein Einfluss auf den $\alpha 1$ Rezeptor nachweisbar war, wurde e in e spezifische Hemmung des bei dem Symptomkomplex der Inkontinenz interessantesten Rezeptors M3, eine IC50 zwischen 3-30 $\mu \mathrm{g} / \mathrm{ml}$ nachgewiesen (Abb. 2). Zudem wurde die Aromatase, welche Testosteron zu Östradiol umwandelt, mit einer IC50 von 70 $\mu \mathrm{g} / \mathrm{ml}$ gehemmt. Der Serotonin-Transporter, zuständig für die SerotoninWiederaufnahmen, wurde mit hoher Affinität bei schon $0.3 \mu \mathrm{g} / \mathrm{m}$ zu $80 \%$ inhibiert.

Auch wenn In-vitroResultate mit Vorsicht interpretiert werden sollten, geben diese Bindungsstudien doch Anhaltspunkte, die sich mit klinischen Erfahrungen, gerade bei Frauen mit Inkontinenzbeschwerden, decken. Um aber gesicherte Daten zu diesen Einsatzgebieten zu erhalten, ist sicher die Durchführung von klinischen Studien mit symptomatischen Patienten nötig.

\section{Schlussfolgerung}

Im Gegensatz zu synthetischen Monosubstanzen, die meist nur gezielt für einen pharmakologischen Wirkort geschaffen wurden, hat man bei pflanzlichen Extrakten den Vorteil, dass es sich um Vielstoffgemische handelt, die an mehreren Wirkorten wirksam sein können. Für den Sägepalmenextrakt A. Vogel ProstaMed ${ }^{\circledR}$ wurde in diesen drei Untersuchungen aufgezeigt, dass neben dem Einsatz zur Behandlung der BPH auch eine antimikrobielle und apoptotische Wirkung besteht und ers- te Anhaltspunkte für den Einsatz bei Inkontinenzbeschwerden bei Frauen bestehen. Eine weitere Erforschung dieser Indikationen, zumal mit klinischen Studien, könnte das Einsatzgebiet dieser interessanten Heilpflanze im Rahmen der rationalen Phytotherapie ermöglichen.

\section{Literatur}

1. Wagner H. : Pharmazeutische Biologie 2: Drogen und ihre Inhaltsstoffe. Gustav Fischer Verlag Stuttgart, New York, 5. Auflage 1993: 348.

2. Hale EM: Saw Palmetto, its History, Botany, Chemistry, Pharmacology, Provings, Clinical Experience and Therapeutic Applications. Soericke \& Tafel, Philadelphia, 1898.

3. Madaus G.: Lehrbuch der biologischen Lehrmittel. Band 111 und Register. Georg Olms Verlag Hildesheim, New York, Auflage 1976.

4. Prager N., Sickett K., French N, Markovich G.: A randomized, double blind, placebo controlled trial to determine the effectiveness of botanically derived inhibitors of 5 alpha-reductase in the treatment of androgenetic alopecia. J Altern Complement Mod 2002;8(2): 143-144.

5. Sinclair RD, Mallari RS., Tate B., : Sensitization to saw palmetto and minoxidil in spearate topical extemporaneous treatments for androgenetic alopecia. Australas J Dermatol 2002; 43(4):311-2.

6. Yang J, Te AE Saw palmetto and finasteride in the treatment of category-III prostatitis/chronic pelvic pain syndrome. Curr Urol Rep. 2005 Jul;6(4):290-5.

\section{Anschrift des Autors:}

Andy Suter, Leiter Medizin

Bioforce AG, CH-9325 Roggwil

A.Suter@bioforce.ch 